\title{
Investigation of Chlamydia trachomatis IgG and Treponema pallidum IgM antibodies among undergraduate female young stars of Niger Delta Extraction
}

\author{
Obioma Azuonwu*, Ossai Jennifer and Christian Serekara \\ Department of Medical Laboratory Science, Faculty of Science, Rivers State University, Port Harcourt, Nigeria
}

\begin{abstract}
We performed a cross sectional study which was aimed at evaluating the prevalence of Chlamydia trachomatis IgG and Treponema pallidum IgM antibodies in the sera of young women in Port Harcourt. Two millilitre of venous blood was drawn and was used to assay for Chlamydia trachomatis IgG and Treponema pallidum IgM antibodies from 205 female subjects, aged between 17-28 years, who was recruited into the study through convenience, random sampling research design. The prevalence of Chlamydia trachomatis IgG antibodies was $7.3 \%$ and Treponema pallidum IgM antibodies was $0.9 \%$. There was a zero prevalence of co-infection. When prevalence was evaluated with stratified age groups, there was a significant difference $(p<0.0001)$ with Chlamydia trachomatis IgG antibodies but not with Treponema pallidum IgM antibodies ( $p=0.0548)$. Increased ages of subject were a predictive risk factor for the acquisition of Chlamydia trachomatis infection, hence, it is recommended that national multicentre study be created with varied platforms and settings, for continuous screening and monitoring of targeted sexually active young women in our communities.
\end{abstract}

\section{Introduction}

Chlamydia and syphilis are sexually transmitted infections of public health concern, especially in developing countries [1], where access to functional health delivery system remains a massive critical challenge. The World Health Organization estimated that 1.3 billion people are suffering from sexually transmitted infections globally with highest rates in regions of America and Africa [2]. One in 20 young people contract a curable STI each year [3].

Young women are less likely to use condoms with their male partners than adults because of lack of access and in many cases young people are not provided with the skills to protect themselves against the risk of infection [4]. Chlamydia and syphilis infections may be as a result of unprotected sex with a good number of short-term partners but may also occur among those who have a long-term unfaithful, partner or husband respectively. Nonetheless, the risk is often greater for those who are in socially and economically marginalized positions as sexual activity may take place within a context of force or violence or in the course of selling sex for a living [2], especially in a probably depressed economy like that of Africa where the statistics of those living in poverty and citizens that are unemployed are geometrically increasing per minute - per minute, all at the detriment of the poor and the weak in the society.

Anatomically, sexually active girls may be at greater risk of contracting STIs than boys because, the cervix and vagina undergo dramatic histological changes due to oestrogen exposure. Hence the cervix in adolescent girls still displays areas of exposed columnar epithelium, a condition known as cervical ectopy [5]. Lack of sex education is one of the reasons why young women are particularly vulnerable to chlamydia and syphilis infection, there is still reluctance in some quarters to acknowledge and properly address adolescent sexual activity despite widespread evidence of how early sex begins [2].
There are myriads of complications that could arise from infections by chlamydia and syphilis. Nevertheless, pelvic inflammatory disease, tubular factor infectivity and tubular pregnancy may probably be linked to asymptomatic complication of chlamydia trachomatis infection, even as the above-mentioned disease conditions may critically promote fast dynamics spread of HIV infection among susceptible cohorts.

Nevertheless, several studies with strong evidence-based outcome have reported the prevalence of these infections in different settings, using different testing formats, thus these has resulted in varied result outcome. However, antibodies-based test validates the present of an infectious agent hence very appropriate for our study [2,3]. However, this study is aimed at evaluating the prevalence of Chlamydia trachomatis IgG and Treponema pallidum IgM antibodies in the sera of young women in Port Harcourt; hence, there is a paucity of epidemiological research information in this direction, in the region. Nevertheless, it is strongly expected that data generated from this present study would promote a strong renewed effort towards prompt diagnosis and better management strategies of the infection among young female in the region, with the overall goal of reducing the prevalence rate of the disease in our communities, even as we are also expecting that government budget and funding towards tackling the infection would be palpable and appreciably significant in action.

${ }^{*}$ Correspondence to: Obioma Azuonwu, Department of Medical Laboratory Science, Rivers State University, Port Harcourt, Nigeria, Tel: +234 8035519688 , E-mail: bimajacobs@yahoo.co.uk

Key words: treponema pallidum, chlamydia trachomatis, immunoglobulins, young female undergraduates, niger delta

Received: December 10, 2019; Accepted: January 04, 2020; Published: January 07,2020 


\section{Material and methods}

\section{Subjects/study population}

A total of 205 apparently healthy young female students of the Rivers State University, Port Harcourt, Nigeria; age between 17-28 years were enrolled into our prospective cross-sectional study, however, convenient random sampling technique was explored during subjects recruitment Inclusion criteria for the study included subjects who are not on any antibiotics for 90 days prior to the collection of samples, and who offered acceptance of being sexually active, while those who could not provide consent to be included were excluded from the research.

\section{Ethical clearance}

Consent and ethical clearance were obtained from all subjects and institutional ethical clearance was duly obtained from the Rivers State Ministry of Health and Rivers State Hospital Management Board ethical committee respectively.

\section{Blood collection}

Blood was collected with by venipuncture, place into $2 \mathrm{ml}$ vaccutainer tubes containing Ethylene Diamine Tetra Acetic acid (EDTA) and transported in ambient temperature to working laboratory.

\section{Experimental- determination of chlamydia IgG antibodies}

Method: Enzyme Immunosorbent Assay ImmunoComb ${ }^{\circledR}$ Orgenics

Principles:Chlamydia trachomatis Immunoglobin G (IgG) test is an indirect solid-phase Enzyme immunoassay. At the onset of the test, plasma specimens are added to the diluent in the wells of row A of the Developing Plate. The Card is then inserted in the wells of row A. Anti-C. trachomatis antibodies, if present in the specimens, will specifically bind to the respective chlamydial antigens on the lower spot of each tooth of the Card. Simultaneously, immunoglobulins present in the specimens will be captured by the anti-human immunoglobulin on the upper spot (Internal Control). Unbound components are washed away in row $B$. In row $C$, the human IgG captured on the teeth will react with alkaline phosphatase (AP)-labeled anti-human IgG. In the next two rows, unbound components are removed by washing. In row $\mathrm{F}$, the bound alkaline phosphatase will react with chromogenic components. The results are visible as gray-blue spots on the surface of the teeth of the Card.

\section{Determination of treponema antibodies}

Method: Chemiluminescent microparticle immunoassay (CMIA)

Principles: The ARCHITECT Syphilis Treponema pallidum assay is a two-step immunoassay for the qualitative detection of antibody to Treponema pallidum in human plasma using CMIA technology with flexible assay protocols, referred to as Chemiflex. In the first step, sample, microparticles coated with recombinant Treponema pallidum antigens (TpN15, TpN17 and TpN47) and Assay Diluent are combined. Anti- Treponema pallidum antibodies present in the sample bind to the Treponema pallidum coated microparticles. After washing, the acridinium-labeled anti-human IgM conjugate is added in the second step. Following another wash cycle, Pre-Trigger and Trigger Solutions are added to the reaction mixture. The resulting chemiluminescent reaction is measured as relative light units (RLUs). A direct relationship exists between the amount of anti- Treponema pallidum antibodies in the sample and the RLUs detected by the ARCHITECT optical system. The presence or absence of anti- Treponema pallidum antibodies in the specimen is determined by comparing the chemiluminescent signal in the reaction to the cutoff signal determined from a previous ARCHITECT
Syphilis Treponema pallidum calibration. If the chemiluminescent signal in the specimen is greater than or equal to the cut-off signal, the specimen is considered reactive for anti- Treponema pallidum.

\section{Data analysis}

Graphpad prism 6.0 statistical software was used to analyse the data generated in this study. Prevalence of markers and co-variate were expressed in percentages. Degree of association between sociodemographic factors and variables was tested with Pearson chi-square. An error probability ( $p$-value) $\leq 0.05$ was considered significant.

\section{Results}

Table 1 show the prevalence of Chlamydia trachomatis IgG and Treponema pallidum IgM antibodies, and the concomitant existence of these antibodies in the sera of the study population. Of the 205 subjects tested, $15(7.3 \%)$ subjects demonstrated Chlamydia trachomatis IgG antibodies whereas $2(0.9 \%)$ subjects demonstrated Treponema pallidum IgM antibodies. There was no concomitant existence of both antibodies in the sera of these subjects.

Table 2 shows the prevalence of Chlamydia trachomatis IgG antibodies in the sera of subjects relative to their age group. There was significant difference in the prevalence of Chlamydia trachomatis IgG antibodies $(p<0.0001)$ relative to these groups.

Table 3 shows the prevalence of Treponema pallidum IgM antibodies in the sera of subjects relative to their age groups. There was no significant difference in the prevalence of Treponema pallidum IgM antibodies $(p=0.0548)$ relative to these groups.

A total of 205 female subjects were recruited into this crosssectional study. The age range of these subjects enrolled into the study was 17-28 years. Other demographic characteristics are cited in table 4

Table 1. Distribution of Chlamydial and Syphilitic antibodies among subjects

\begin{tabular}{|c|c|c|c|c|c|c|}
\hline \multirow{2}{*}{ Age } & \multicolumn{2}{|c|}{ Chlamydial IgG } & \multicolumn{2}{c|}{ Treponema IgM } & \multicolumn{2}{c|}{ Coinfection } \\
\cline { 2 - 7 } & No Pos & \% Pos & No Pos & \% Pos & No Pos & \% Pos \\
\hline $17-28$ & 15 & 7.3 & 2 & 0.9 & 0 & 0 \\
\hline Total & 15 & 7.3 & 2 & 0.9 & 0 & 0 \\
\hline
\end{tabular}

Table 2. Prevalence of Chlamydia IgG antibodies according to age of subjects

\begin{tabular}{|c|c|c|c|}
\hline Age Range & Positive (\%) & Negative (\%) & $\chi^{2}$ (P-value) \\
\hline $17-19$ & $0(0 \%)$ & $61(100 \%)$ & $22.21(<0.0001)$ \\
\hline $20-22$ & $0(0 \%)$ & $51(100 \%)$ & \\
\hline $23-25$ & $6(12 \%)$ & $44(88 \%)$ & \\
\hline $26-28$ & $9(21 \%)$ & $34(79 \%)$ & \\
\hline
\end{tabular}

Table 3. Prevalence of Treponema IgM antibodies according to age of subjects

\begin{tabular}{|c|c|c|c|}
\hline Age Range & Positive (\%) & Negative (\%) & $\chi^{2}$ (P-value) \\
\hline $17-19$ & $0(0 \%)$ & $61(100 \%)$ & $7.609(0.0548)$ \\
\hline $20-22$ & $0(0 \%)$ & $51(100 \%)$ & \\
\hline $23-25$ & $0(0 \%)$ & $50(100 \%)$ & \\
\hline $26-28$ & $2(0.9 \%)$ & $41(99.1 \%)$ & \\
\hline
\end{tabular}

Table 4. Demographic Characteristics of Enrolled Subjects

\begin{tabular}{|l|c|}
\hline Features & Statistics \\
\hline Total No. of subjects & 205 \\
\hline Age Range (Years) & $17-28$ \\
Age Grouping (Relative Number): & \\
$17-19$ & 61 \\
$20-22$ & 51 \\
$23-25$ & 50 \\
$26-28$ & 43 \\
\hline
\end{tabular}




\section{Discussion}

This study was aimed at evaluating the prevalence of Chlamydia trachomatis IgG and Treponema pallidum IgM antibodies in the sera of young women in Port Harcourt.

An estimated 1.3 billion people are suffering from sexually transmitted diseases globally [2]. These infections include those caused by Chlamydia trachomatis and Treponema pallidum, which are also an emerging public health concern globally and, especially in developing countries [1]. In sub Saharan Africa, 1 in 20 young people contract a curable STI each year [3].

The prevalence of Chlamydia trachomatis IgG antibodies depends to some extent on the population of study and the diagnostics performance of the screening method used [6]. In our study, the prevalence of Chlamydia trachomatis IgG antibodies in study population was 7.3 percent. This is at variance with the report of other researchers in Nigeria. In an earlier study conducted by Obunge et al. [7] in Port Harcourt on the prevalence of C. trachomatis among adolescent females, they obtained a prevalence of $37.5 \%$. Other studies had also obtained variable results, such as, Jeremiah et al. [8] obtained a prevalence of $51 \%$ among apparently healthy pregnant women in Port Harcourt. In another study investigating the prevalence of $C$. trachomatis among younger women in Port Harcourt, Wariso et al. [9] obtained a prevalence of $11 \%$. The reason for these disparities could be difference in subject's population and settings, sociodemographic, and testing methods.

Nevertheless, in our study, behavioural risk stratification was not adopted while interacting with the subjects in other to determine sexual behaviours aside from age and a positive consent of being sexually active. The lower prevalence observed for Chlamydia trachomatis IgG antibodies in this study might indicate relative reduction in this infection in the population since serum antibody formation mirror of only initial exposure to a specific antigen.

The prevalence of Treponema pallidum in the general population present yet discordance outcome [10]. Our study demonstrated a prevalence of $0.9 \%$ for Treponema pallidum IgM antibodies in the sera of young undergraduate females in Port Harcourt (Table 2,4). This figure is lower than $2.9 \%$ obtained by Lindstrand et al. [11], while working in Oshogbo, Southwestern Nigeria. Aboyeji and Nwabuisi [12] had a prevalence of $1.7 \%$ in their work in Ilorin.

Studies from other countries had reported varied results, In Zambia, Todd CS [13] reported a prevalence of $12.5 \%$ among pregnant women; Lindstrand [11] found $18.3 \%$ among antenatal care attendees in Mozambique. In Malawi, obtained 5\% prevalence among pregnant women. However, no case of syphilis infection was found in the 4,452 pregnant Afghanistan women receiving antenatal care at three Government maternity hospitals in Kabul [13].

Observed differences in the prevalence of Treponema pallidum syphilis infection in the different populations of women within and outside Nigeria might be a reflection of the variation in sexual practice and behaviour of the societies where the studies were carried out. It may also be due to demographics variation, difference in accessibility to treatment of STIs, cultural practices, and differences in the diagnostic characteristics of sensitivity, specificity and predictive values of methods adopted for testing, and the current societal awareness on prevention of STIs.

There is a paucity of information with regards to Chlamydia and Syphilis co-infection. Our study demonstrated zero prevalence for this co-infection (Table 1). Increase age of women had been a predictive risk factor aside past history of STIs, multiple sexual partners and poor compliance to use of protective barriers [9].

In comparing the effect of age to the prevalence of Chlamydia trachomatis IgG and Treponema pallidum IgM antibodies in the sera of subjects, we found statistically significant difference $(<0.0001)$ between the age groups, with age group 26-28 years having the highest prevalence for Chlamydia trachomatis IgG antibodies (Table 4). The higher prevalence recorded for these age groups could be due to long exposure of this group to different sexual activities. Treponema pallidum IgM antibodies in the sera of subjects did not shows age group discrimination $(p=0.0548)$ (Table 4$)$.

Nonetheless, it has been extremely proven to be very cumbersome to at rightly identify possible risk factors in a given population with high degree of sensitivity and specificity, hence universal testing of sexually active young female population should be targeted from time to time. Nevertheless, the high propensity at which those vulnerable are infected are strong indication that women who are sexually active, should be universally tested, every 6 monthly to rule out or reduce the continuous spread and the possibilities of re-infection among the already diagnosed and treated subjects in our communities.

\section{Conclusion}

The established prevalence of Chlamydia trachomatis IgG and Treponema pallidum IgM antibodies in the sera of young undergraduate female students in Port Harcourt was lower than those of other studies. No co-infection between Chlamydia trachomatis IgG and Treponema pallidum IgM antibodies was seen among the study population. Increased ages of subject were a predictive risk factor for the acquisition of Chlamydia trachomatis infection.

\section{Acknowledgement}

The researchers are profoundly grateful to the participants for volunteering to be part of the study, even as we are thankful to the laboratory staff of the Rivers State Health Services Department for their immense support during the study. We are also grateful to Prof Wokem G.N, Prof S D Abbey and Dr Azuonwu Goodluck for their unreserved prayers and support.

\section{Conflict of interest}

None reported among authors.

\section{References}

1. Msuya SE, Uriyo J, Hussain A, Mbizvo EM, Jeansson S, et al. (2010) Prevalence of sexually transmitted infections among pregnant women with known HIV status in northern Tanzania. Reproductive Health 6: 4. [Crossref]

2. WHO (2013) Sexually Transmitted Infections (STIs): The Importance of a Renewed Commitment to STI Prevention and Control in Achieving Global Sexual and Reproductive Health. Available at: https://www.google.com/search?q=WHO.+(2013).+ $\%$ E2\% $\% 0 \% 9$ CSexually+Transmitted+Infections $+(\mathrm{STIs}) \% 3 \mathrm{~A}+$ The+Importance + of + a + Renewed+Commitment+to+STI+Prevention+and+Control+in+Achieving+Global+ Sexual + and + Reproductive + Health\&oq $=$ WHO. $+(2013) .+\%$ E $2 \% 80 \% 9$ CSexually + Tra nsmitted + Infections $+($ STIs) $\% 3 \mathrm{~A}+$ The + Importance + of $+\mathrm{a}+$ Renewed + Commitment $+\mathrm{t}$ $\mathrm{o}+\mathrm{STI}+$ Prevention + and + Control + in + Achieving + Global + Sexual + and + Reproductive + Health\&aqs $=$ chrome..69i57.4017j0j7\&sourceid $=$ chrome\&ie $=$ UTF-8 $\left[\right.$ Accessed $5^{\text {th }}$ of December, 2019]

3. WHO (2008) Global incidence and prevalence of selected curable sexually transmitted infections. Available at: https://www.google.com/search?q=WHO.+(2008)\%2C+\%E2 $\% 80 \% 9$ CGlobal+incidence+and + prevalence + of + selected + curable + sexually+transmitt ed+infections. $\%$ E2\% $\% 0 \% 9$ D\&oq=WHO. $+(2008) \% 2 \mathrm{C}++\% \mathrm{E} 2 \% 80 \% 9$ CGlobal+incide nce+and+prevalence + of + selected + curable + sexually + transmitted + infections. $\% \mathrm{E} 2 \% 80$ $\% 9$ D\&aqs $=$ chrome..69i57.7412j0j9\&sourceid $=$ chrome \&ie $=$ UTF-8 
4. Eaton DK, Kann L, Kinchen S (2012) Youth risk behavior surveillance. Morbidity and mortality weekly report surveillance summaries 61: 1-162.

5. Cheng, SY, Lo KK (2012) Sexually transmitted infections in adolescents. Hong Kong Journal of Paediatrics 7: 76-84.

6. Malhotra M, Bala M, Muralidhar S, Khunger N, Puri P (2008) Prevalence of Chlamydia trachomatis and its association with other sexually transmitted infection in tertiary care centre in North India. Indian Journal of Sexually Transmitted Diseases and AIDS 29 . $82-85$.

7. Obunge OK, Brabin L, Dollimore N, Kemp J, Ikokwu-Wonodi C (2001) A Flowchar for Managing Sexually Transmitted Infection among Nigerian Adolescent Females. Bulletin of the World Health Organization 79: 301-305. [Crossref]

8. Jeremiah I, Okeke O, Akani C (2011) The Prevalence of Serum Immunoglobulin G antibody to Chlamydia Trachomatis in Subfertile Women Presenting at the University of Port Harcourt Teaching Hospital, Nigeria. International Journal of Biomedical Science 7: 120-124. [Crossref]
9. Wariso KT, Odigie J, Eyaru S (2012) Prevalence of Chlamydia Trachomatis infection among female undergraduates of University of Port Harcourt using Strand Displacement and Amplification Techniques. Nigerian Health Journal 12: 35-38. [Crossref]

10. Ogiogwa, JI, Akingbade OA, Aboderin BW, Okerentugba PO, Innocent-Adiele HC, et al. (2012) Prevalence of Treponema pallidum Serum of Pregnant Women in Abeokuta, Ogun State, Nigeria. Nature and Science 10: 91-94.

11. Lindstrand A, Bergstrorm S, Bugalho A, Zanconato G, Helgesson AM, et al. (1993) Prevalence of syphilis infection in Mozambican women with second trimester miscarriage and women attending antenatal care in second trimester. Genitourinary Medicine 9: 421-433. [Crossref]

12. Aboyeji AP, Nwabuisi C (2003) Prevalence of sexually transmitted disease among pregnant women in Ilorin, Nigeria. Journal of Obstetrics and Gynaecology 23: 637639. [Crossref]

13. Todd CS, Ahmdzai M, Atiqzai F, Miller S, Smith JM, et al. (2008) Seroporevalence and correlate of HIV, Syphilis, and hepatitis B and C virus among intrapartum patients in Kabul, Afghanistan. Infectious Diseases 8: 119. [Crossref]

Copyright: $(02020$ Azuonwu O. This is an open-access article distributed under the terms of the Creative Commons Attribution License, which permits unrestricted use, distribution, and reproduction in any medium, provided the original author and source are credited. 